\title{
Noisy Relativistic Quantum Games in Noninertial Frames
}

\author{
Salman Khan ${ }^{\dagger *}$ M. Khalid Khan ${ }^{\ddagger}$ \\ ${ }^{\dagger}$ Department of Physics, COMSATS Institute of Information \\ Technology, Islamabad 44000, Pakistan. \\ ${ }^{\ddagger}$ Department of Physics, Quaid-i-Azam University, \\ Islamabad 45320, Pakistan.
}

September 26, 2018

\begin{abstract}
The influence of noise and of Unruh effect on quantum Prisoners' dilemma is investigated both for entangled and unentangled initial states. The noise is incorporated through amplitude damping channel. For unentangled initial state, the decoherence compensates for the adverse effect of acceleration of the frame and the effect of acceleration becomes irrelevant provided the game is fully decohered. It is shown that the inertial player always out scores the noninertial player by choosing defection. For maximally entangled initially state, we show that for fully decohered case every strategy profile results in either of the two possible equilibrium outcomes. Two of the four possible strategy profiles become Pareto Optimal and Nash equilibrium and no dilemma is leftover. It is shown that other equilibrium points emerge for different region of values of decoherence parameter that are either Pareto optimal or Pareto inefficient in the quantum strategic spaces. It is shown that the Eisert et al 2 miracle move is a special move that leads always to distinguishable results compare to other moves. We show that the dilemma like situation is resolved in favor of one player or the other.

PACS: 02.50.Le, 03.67.Bg,03.67.Ac, 03.65.Aa.
\end{abstract}

Keywords: Quantum games;Decoherence: Unruh effect; Noninertial frames

\section{Introduction}

Quantum game theory is a study of the well established field of classical game theory in the light of the principals of quantum mechanics. It exploits the remarkable properties of quantum mechanics, the entanglement and the quantum phases, to get results that are classically impossible. For a little more than one

*sksafi@phys.qau.edu.pk 
decade, quantum game theorists are involved to study the behavior of classical games in the domain of quantum mechanics under different circumstances. A number of classical games have been quantized and the effects of entanglement on the payoff of the players have been studied. Subsequent to the work of Meyer [1, it has been shown by many authors that quantum players can outsmart the classical counterparts by using quantum mechanical strategies [2-13].

The entanglement between spatially separated parties is the mere powerful source of performing various quantum information and quantum computation processes. Its behavior in relativistic setup in noninertial frames is presently under exploration. The existing studies in the noninertial frames show that the Unruh effect degrades the entanglement between different modes of various fields that may or may not vanish in the limit of infinite acceleration 14, 15, 16, 17, 18, 19. The studies of the effect of environment on entanglement in noninertial frames [20, 21, 22 shows that the decoherence quickens the loss of entanglement in many cases, nevertheless, under particular conditions the entanglement rebirth may happen [20].

In this paper, we study the effect of environment and of Unruh effect on the payoffs function of the players in the quantum Prisoners' Dilemma [2. The effect of environment is incorporated using amplitude damping noise. We show that for a factorizable initial state and fully decohered game, the acceleration of the frame become irrelevant and the payoff matrix reduces to the classical symmetric payoff matrix. The local environment of stationary observer has no effect on the payoff function of the players. For maximally entangled initial state, the strategy profiles $(\hat{C}, \hat{C})$ and $(\hat{D}, \hat{D})$ become Pareto inefficient when the game is fully decohered. We show that in the presence of noise, the best strategy to the classical strategy $\hat{D}$ is the quantum strategy $\hat{Q}$ (see Eq. (15)). The strategy profile $(\hat{Q}, \hat{C})$ is Pareto inefficient and the strategy profile $(\hat{Q}, \hat{Q})$ is Pareto optimal as well as Nash equilibrium. We point out the origin of inconsistency between the results of [23] and [2] and show that in the noiseless relativistic game, the miracle move of Eisert et al 2] is a game winning move. Depending on the level of noise, we show that the dilemma can be completely or partially resolved.

\section{The Prisoners' Dilemma}

The classical Prisoners' Dilemma is a two player non-zero sum game. The strategic space of each player consists of two strategies, cooperation $(C)$ and defection $(D)$. The players (Alice and Bob) are supposed to choose a move from their respective strategic spaces simultaneously. The reward to the action of a player depends not only on his own move but also on the move of his opponent. The rewards to all the possible strategic profiles of the game are shown in the payoff matrix in Table 1. The left number in each pair of the matrix represents Alice's payoff and the right number in a pair stands for Bob's payoff. This is a symmetric noncooperative game where each player tries to maximize his/her own payoff. The catch of the dilemma is that $D$ is the dominant strategy, that is, 
rational reasoning forces each player to defect, and thereby doing substantially worse than if they would both decide to cooperate. The behavior of the game in the domain of quantum mechanics were first studied by Eisert et al [2]. The influence of Unruh effect on the payoffs of the players in quantum prisoners' dilemma in the relativistic setup is recently studied in Ref. [23].

Table 1: Payoff matrix for the classical Prisoners' Dilemma. The first entry in a pair of numbers denotes the payoff of Alice and the second entry represents Bob's payoff.

\begin{tabular}{|c|c|c|}
\hline \hline & Bob: $C$ & Bob: $D$ \\
\hline \hline Alice: $C$ & 3,3 & 0,5 \\
\hline \hline Alice: $D$ & 5,0 & 1,1 \\
\hline \hline
\end{tabular}

\section{Calculation}

We consider that Alice and Bob share an entangled initial state $\left|\psi_{i}\right\rangle_{M}=\hat{J}\left|0_{\omega_{A}}\right\rangle_{M}\left|0_{\omega_{B}}\right\rangle_{M}$ of two fermionic qubits of mode frequencies $\omega_{A}$ and $\omega_{B}$ at a point in flat Minkowski spacetime and all the other modes are in vacuum state from the perspective of the inertial observer. The subscript $M$ of the kets specifies the Minkowski spacetime. The first ket is in Alice possession and the second ket is in Bob possession. The operator $\hat{J}$ is a symmetric unitary operator that works as an entangling gate and is known to both players. Mathematically, it is given by

$$
\hat{J}=\exp \left[i \frac{\gamma}{2} \hat{D} \otimes \hat{D}\right]
$$

where $\gamma \in[0, \pi / 2]$ and is a measure of the degree of entanglement in the initial state. The initial state has no entanglement for the lower limit of $\gamma$ and is maximally entangled for the upper limit of $\gamma$. The operator $\hat{D}$ is given by

$$
\hat{D}=\left(\begin{array}{cc}
0 & i \\
i & 0
\end{array}\right)
$$

The initial state, after the entangling operator is applied, becomes

$$
\left|\psi_{i}\right\rangle_{M}=\cos \frac{\gamma}{2}\left|0_{\omega_{A}}\right\rangle_{M}\left|0_{\omega_{B}}\right\rangle_{M}-i \sin \frac{\gamma}{2}\left|1_{\omega_{A}}\right\rangle_{M}\left|1_{\omega_{B}}\right\rangle_{M}
$$

In Eq. (3), $\left|0_{\omega_{N}}\right\rangle$ and $\left|1_{\omega_{N}}\right\rangle(N=A, B)$ kets represent the vacuum and the excited states from the perspective of an inertial observer. We consider that both the players carry devices that are sensitive to their respective modes $\omega_{N}$. After sharing these modes, Bob then moves with a uniform acceleration and Alice stays stationary. The suitable coordinates for accelerated observers are Rindler coordinates, which define two causally disconnected Rindler regions $(I, I I)$. That is, a uniformly accelerated observer in region $I$ has no access 
to information that leaks to region $I I$ and vice versa (for detail see [14] and reference therein). A given Minkowski mode of a particular frequency spreads over all positive Rindler frequencies that peaks about the Minkowski frequency 24, 25]. However, to simplify our problem we consider a single mode only in the Rindler region $I$, which is valid if the observers' detectors are highly monochromatic that detects the frequency $\omega_{A} \sim \omega_{B}=\omega$. From this point onward, with this approximation, the frequency subscript of kets will be dropped.

From the perspective of accelerated frame, the Minkowski vacuum state is a two mode squeezed state given by [14, 26, 27, 28]

$$
|0\rangle_{M}=\cos r|0\rangle_{I}|0\rangle_{I I}+\sin r|1\rangle_{I}|1\rangle_{I I},
$$

and the Minkowski excited state is given by

$$
|1\rangle_{M}=|1\rangle_{I}|0\rangle_{I I},
$$

where $I$ and $I I$ in the subscript of the kets represents the modes in the two Rindler regions. Eq. (4) shows that the noninertial observer that moves with a constant acceleration in region $I$ sees a thermal state instead of the vacuum state. This effect is called the Unruh effect [29, 30]. The parameter $r$ is the

dimensionless acceleration parameter given by $\cos r=\left(e^{-2 \pi \omega c / a}+1\right)^{-1 / 2}$. The constants $\omega, c$ and $a$, in the exponential stand, respectively, for Dirac particle's frequency, speed of light in vacuum and Bob's acceleration. The parameter $r=0$ when acceleration $a=0$ and $r=\pi / 4$ when $a=\infty$. Using Eqs. (4) and (5) in Eq. (3), the initial state in terms of Minkowski mode for Alice and Rindler modes for Bob becomes

$$
\begin{aligned}
|\psi\rangle_{M, I, I I}= & \cos \frac{\gamma}{2} \cos r|0\rangle_{M}|0\rangle_{I}|0\rangle_{I I} \\
& +\cos \frac{\gamma}{2} \sin r|0\rangle_{M}|1\rangle_{I}|1\rangle_{I I}-i \sin \frac{\gamma}{2}|1\rangle_{M}|1\rangle_{I}|0\rangle_{I I} .
\end{aligned}
$$

Since Bob has no access to the information in region $I I$, therefore, tracing over the modes in region $I I$ gives the following mixed density matrix

$$
\begin{aligned}
\rho_{M, I}= & \cos ^{2} \frac{\gamma}{2} \cos ^{2} r|00\rangle\left\langle 00\left|-\frac{1}{2} i \sin \gamma \cos r\right| 11\right\rangle\langle 00| \\
& +\cos ^{2} \frac{\gamma}{2} \sin ^{2} r|01\rangle\left\langle 01\left|+\frac{1}{2} i \sin \gamma \cos r\right| 00\right\rangle\langle 11| \\
& +\sin ^{2} \frac{\gamma}{2}|11\rangle\langle 11| .
\end{aligned}
$$

Note that we have dropped the subscript of the kets.

\subsection{The game in a noisy environment}

Now, we consider that prior to the execution of moves by the players, the state of the game evolves through a noisy environment. The interaction of a quantum system with a noisy environment can be described in terms of Kraus operators. 
The final density matrix of a system when it evolves in a noisy environment can be written as

$$
\rho_{f}=\sum_{i, j, k, \ldots} K_{i} K_{j} K_{k} \ldots \rho \ldots K_{k}^{\dagger} K_{j}^{\dagger} K_{i}^{\dagger},
$$

where $\rho$ is the initial density matrix of a system and $K_{n}$ are the Kraus operators that describe the interaction of the system with the environment and satisfy the completeness relation $\sum_{n} K_{n}^{\dagger} K_{n}=I$. There could be different kinds of interactions between the system and environment and each kind of interaction has its own form of Kraus operators. The kind of interaction that we consider here is known as amplitude damping. The Kraus operators for amplitude damping channel of a single qubit system are given as

$$
K_{o}=\left(\begin{array}{cc}
1 & 0 \\
0 & \sqrt{1-p}
\end{array}\right), \quad K_{1}=\left(\begin{array}{cc}
0 & 0 \\
0 & \sqrt{p}
\end{array}\right)
$$

where $p(0 \leq p \leq 1)$ is called decoherence parameter. The system is undecohered for the lower limit and is fully decohered for the upper limit of $p$. In the following we consider two kinds of interactions. In one case, we study the coupling of only Bob's qubit with the environment and in the second case both the qubits are under the influence of the environment.

The Kraus operators $E_{i}$ for the case when only Bob's qubit is locally under the influence of environment can be expressed in terms of the single qubit Kraus operators as $E_{i}^{B}=I \otimes K_{i}$, where $I$ is a single qubit identity matrix and $i=0,1$. Similarly, when Alice's qubit is locally influenced by the environment, the Kraus operators for that case become $E_{i}^{A}=K_{i} \otimes I$. The density matrix of the system, after the coupling with environment, becomes

$$
\rho_{M, I, E}=\sum_{i} E_{i}^{A} E_{i}^{B} \rho_{M, I} E_{i}^{B \dagger} E_{i}^{A \dagger},
$$

where $E$ in the subscript of $\rho$ is a reminiscence that the game state is influenced by the environment. The decoherence parameter for Alice's qubit is represented by $p_{1}$ and for Bob's qubit it is given by $p_{2}$.

In the quantum Prisoners' Dilemma, the strategic moves of the players are unitary operators that can be expressed as 32

$$
\hat{U}_{N}(\alpha, \theta)=\left(\begin{array}{cc}
e^{i \alpha_{N}} \cos \frac{\theta_{N}}{2} & i \sin \frac{\theta_{N}}{2} \\
i \sin \frac{\theta_{N}}{2} & e^{-i \alpha_{N}} \cos \frac{\theta_{N}}{2}
\end{array}\right)
$$

where the subscript $N=A, B$ stands for Alice and Bob, $\theta \in[0, \pi]$ and $\alpha \in$ $[-\pi, \pi]$. More generally, the quantum mechanical strategic spaces of the players can be expressed in terms of unitary operators made up of three parameters [31, 32. Nevertheless, in the present work we will use the two parameter strategic spaces of Eq. (11). If the move cooperation of the players is associated with state $|0\rangle$ and the move defection is associated with state $|1\rangle$, then the quantum strategy $\hat{C}$ corresponds to $\hat{U}_{N}(0,0)$ and the quantum strategy $\hat{D}$ corresponds 
to $\hat{U}_{N}(0, \pi)$. After executing their moves, the final density matrix of the game prior to the measurement becomes [2]

$$
\rho=\hat{J}^{\dagger}\left(\hat{U}_{A} \otimes \hat{U}_{B}\right) \rho_{M, I, E}\left(\hat{U}_{A}^{\dagger} \otimes \hat{U}_{B}^{\dagger}\right) \hat{J},
$$

where the disentangling gate $\hat{J}^{\dagger}$ is applied to disentangle the final density matrix in order to make measurement of the payoffs. The expected payoffs of the players are then found by using the following equation

$$
P_{N}^{j_{1} j_{2}}=\sum_{i} \$_{N}^{j_{1(i)} j_{2(i)}} \rho_{i i},
$$

where $\rho_{i i}(i \in[0,1])$ are the diagonal elements of the final density matrix and $\$_{N}^{j_{1}(i) j_{2}(i)}\left(j_{1(i)}, j_{2(i)} \in[C, D]\right)$ are the classical payoffs of the players from table 1 whereas $j_{1}, j_{2}$ in the superscript of $P$ could be any of the classical or quantum strategies.

\section{Results and Discussion}

The payoffs for initially unentangled state $(\gamma=0)$ when the players are restricted to the classical strategic spaces are given in table 2 . In each cell of the table, the top payoff corresponds to Alice and the bottom one corresponds to Bob. It can easily be seen from the table that the results of Ref. [23. are retrieved for $p_{2}=0$ and for $p_{2}=r=0$, the classical results are obtained. Generally, the symmetry of payoff matrix that exists in the classical form of the game is lost and non of the strategy profiles results in same payoffs to both players. However, regarding decoherence, there are two other important features of the payoff matrix of the table that need to be pointed out, (a) the payoffs depend only on the decoherence parameter $p_{2}$, which means that for unentangled initial state the coupling of the stationary player with the environment have no effect on the payoffs of the players. This is an important result, as for practical applications of the game, one doesn't need to bother about the local coupling of the inertial player with the environment. (b) each payoff changes by an amount $\pm n p_{2} \sin ^{2} r$, where $n$ is an integer, such that for a fully decohered $\left(p_{2}=1\right)$ case the acceleration parameter $r$ becomes irrelevant and the symmetric classical payoff matrix is retrieved. For a fully decohered case, the decoherence compensates for the asymmetry of the payoff matrix caused by the acceleration of the frame and brings fairness back to the game. Although,for any sets of values of $p_{2}$ and $r$, Alice dominates by playing $\hat{D}$, however, the dilemma of the classical game exists as the strategy profiles $(\hat{C}, \hat{C})$ and $(\hat{D}, \hat{D})$ are respectively Pareto optimal and Nash equilibrium.

Now, we investigate the effects of decoherence on payoff functions of the players for maximally entangled initial state $(\gamma=\pi / 2)$ by restricting the players first to the classical strategic spaces and then allowing the players to choose their moves from quantum mechanical strategic spaces as well. For both of these 
Table 2: The payoff matrix of the players' payoffs as a function of the acceleration of Bob's frame and decoherence parameter. The top entry in every cell corresponds to Alice's payoff and the bottom entry corresponds to Bob's payoff. The initial state of the game is unentangled and the players are allowed to select a move from the two pure classical moves.

\begin{tabular}{|c|c|c|}
\hline & Bob: $\hat{C}$ & Bob: $\hat{D}$ \\
\hline Alice: $\hat{C}$ & $3\left(\cos ^{2} r+p_{2} \sin ^{2} r\right)$, & $3\left(1-p_{2}\right) \sin ^{2} r$, \\
& $4-\cos 2 r-2 p_{2} \sin ^{2} r$ & $4+\cos 2 r+2 p_{2} \sin ^{2} r$ \\
\hline Alice: $\hat{D}$ & $3+2 \cos 2 r+4 p_{2} \sin ^{2} r$, & $3-2 \cos 2 r-4 p_{2} \sin ^{2} r$, \\
& $\left(1-p_{2}\right) \sin ^{2} r$ & $\cos ^{2} r+p_{2} \sin ^{2} r$ \\
\hline
\end{tabular}

cases, the payoff matrices are very interesting and the results are drastically different from the payoff matrix of the unentangled initial state. We begin for the case when both players are bound to the classical strategic spaces. The payoffs for all the four possible strategy profiles become

$$
\begin{aligned}
P_{A, B}^{C C(D D)}= & \left.\frac{17}{8}+\frac{p_{1}}{4} \pm \sqrt{\left(1-p_{1}\right)\left(1-p_{2}\right)}\right] \cos r-\frac{1}{8} \cos 2 r \\
& +p_{2}\left(\frac{1}{8}-\frac{p_{1}}{2}+\frac{1}{8} \cos 2 r\right), \\
P_{A}^{C D(D C)=} & \left.P_{B}^{D C(C D)}=\frac{19}{8}-\frac{p_{1}}{4} \mp \frac{5}{2} \sqrt{\left(1-p_{1}\right)\left(1-p_{2}\right)}\right] \cos r+\frac{1}{8} \cos 2 r \\
& -p_{2}\left(\frac{1}{8}-\frac{p_{1}}{2}+\frac{1}{8} \cos 2 r\right),
\end{aligned}
$$

where the upper sign in" \pm " and in " $\mp "$ corresponds to the first strategy profile and the lower sign corresponds to the second strategy profile, bracketed in the superscript of each payoff function. For strategy profiles $(\hat{C}, \hat{C})$ and $(\hat{D}, \hat{D})$, the results of Refs. 22, 23] are retrieved by setting, respectively, the corresponding parameters equal to zero. For strategy profile $(\hat{C}, \hat{D})$ and $(\hat{D}, \hat{C})$, setting $p_{i^{\prime}} s=0$, the results are inverted to the results obtained in [23, however, these are in agreement with the results of Ref. 2] for $p_{i^{\prime}} s=r=0$. The inversion of payoffs between the players for strategy profiles $(\hat{C}, \hat{D})$ and $(\hat{D}, \hat{C})$ in 23] happens because of the use of non-symetric form 31, 32 of operator $\hat{D}_{1}$ (Eq. (2)) for constructing the entangling operator $\hat{J}$. The choice of operator $\hat{D}$, in the present work, for constructing the entangling operator $\hat{J}$ removes this mismatch. Unlike the payoff matrix for unentangled initial state (table 2), the payoff matrix of Eq. (14) is a symmetric payoff matrix and each payoff depends on coupling with the two local environments. Furthermore, the strategy profiles $(\hat{C}, \hat{C})$ and $(\hat{D}, \hat{D})$ define two different equilibrium outcomes, like the undecohered case of the game in noninertial frames. Since the payoffs become functions of decoherence parameters $p_{i^{\prime}} s$, we want to see how the payoffs vary with these parameters by considering the simplest case with $p_{1}=p_{2}=p$. This means that instead of two different local environments, both qubits are coupled together to a single collective environment. The payoffs for different strategy 

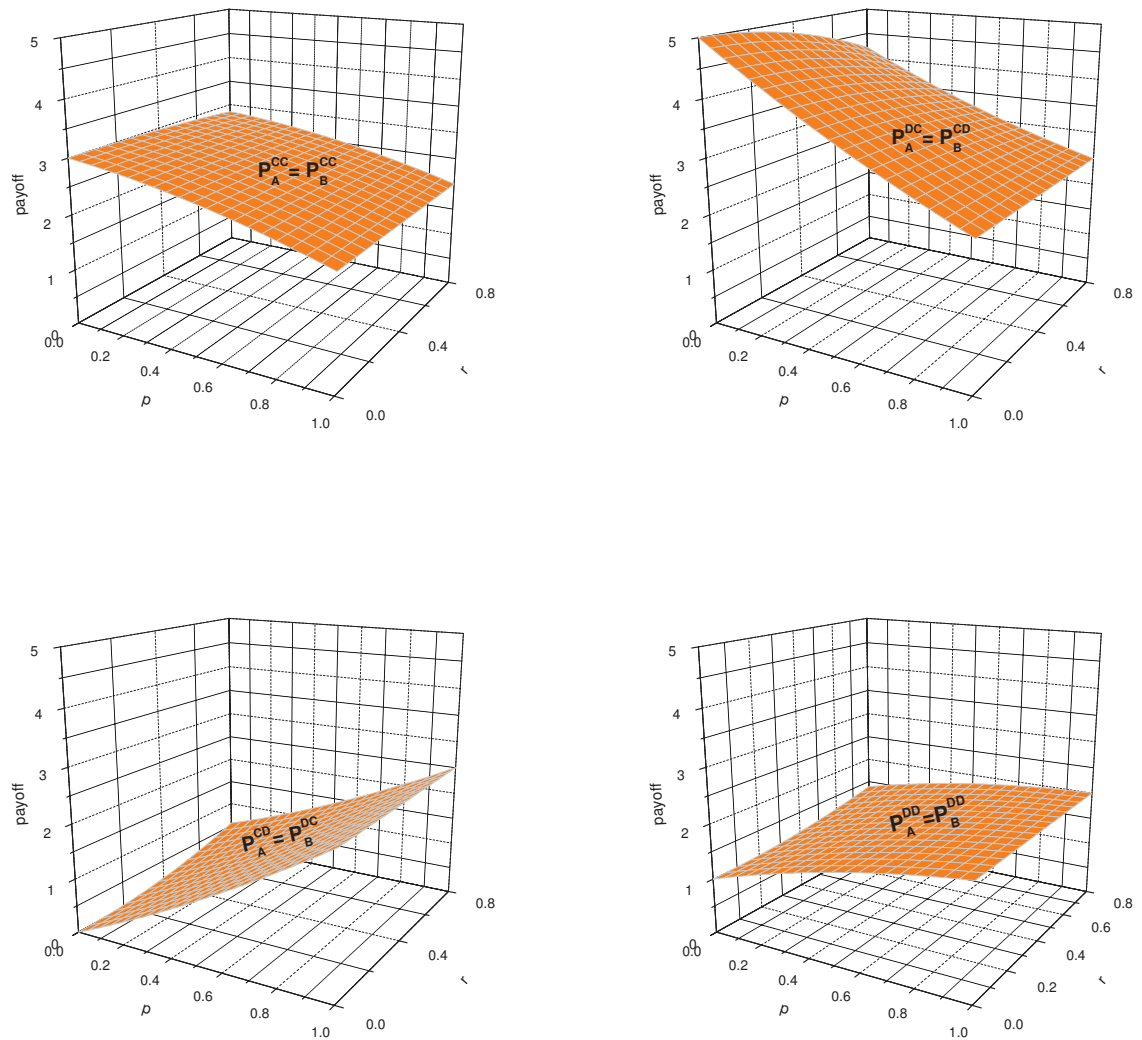

Figure 1: (color online) The payoffs for different strategy profiles are plotted against the decoherence parameter $p$ and the acceleration parameter $r$ for the case when the initially state is maximally entangled.

profiles are plotted against the decoherence parameter $p$ and the acceleration parameter $r$ in figure 1. One can see from the figure that $\hat{D}$ is the dominant strategy. Again, this result is not in agreement with the result of 23 for the same reason as mentioned above. The effect of decoherence is not identical for every strategy profile rather it's somewhat additive to the effect of acceleration parameter $r$. For example, in the first row of figure 1 both $p$ and $r$ reduce the payoffs whereas in the second row both act to increase the payoffs keeping one or the other parameter constant. Also, it is clear from the figure that the strategy profile $(\hat{C}, \hat{C})$ becomes Pareto Optimal and the strategy profile $(\hat{D}, \hat{D})$ becomes Nash equilibrium. This is true for the whole range of decoherence parameter $p$ in the region of lower values of the acceleration of the accelerated frame. In the 
range of infinite acceleration and upper values of $p$ the behavior of the game changes. This change in the behavior of the game is shown in figure 2, where payoffs of Eq. (14), corresponding to different strategy profiles, are plotted against decoherence parameter for $r=\pi / 4$. It can be seen from the figure that, although, $\hat{D}$ is still the dominant strategy, however, for $0.85<p<1$, the payoffs corresponding to the two equilibrium strategy profiles $((\hat{C}, \hat{C})$ and $(\hat{D}, \hat{D}))$ are no good for any player. The most striking feature of figure 2 corresponds to the fully decohered game $(p=1)$ where every strategy profile results into one of the two outcomes. One can see that the strategy profiles $(\hat{C}, \hat{C})$ and $(\hat{D}, \hat{D})$ become Pareto inefficient whereas the strategy profiles $(\hat{C}, \hat{D})$ and $(\hat{D}, \hat{C})$ are both Pareto Optimal and Nash equilibrium and there is no dilemma left in the game. Regardless of the move of a player, the game ends up in either of the two outcomes.

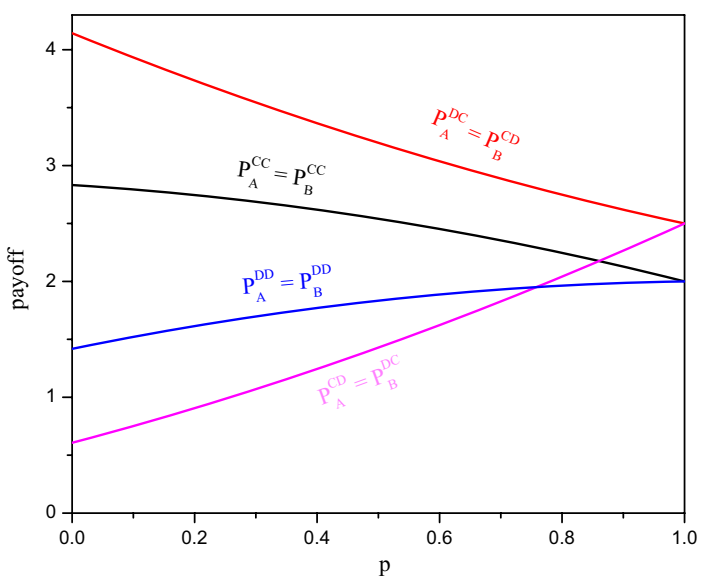

Figure 2: (color online) The payoffs for different strategy profiles are plotted against the decoherence parameter $p$ for the case when the initially state is maximally entangled and the acceleration parameter $r=\pi / 4$.

Now, we turn to the case where players have access to the quantum mechanical strategic spaces. At this end, we first study the effects of decoherence on the payoff function of the players when one or both of them plays the Eisert et 
al. 2] quantum mechanical strategy $\hat{Q}$ given by

$$
\hat{Q}=\hat{U}(\pi / 2,0)=\left(\begin{array}{cc}
i & 0 \\
0 & -i
\end{array}\right)
$$

Let Alice play $\hat{Q}$, then, the payoffs as function of the remaining four parameters $\left(p, r, \alpha_{B}, \theta_{B}\right)$ are given by

$$
\begin{aligned}
P_{A, B}^{Q \theta_{B}}= & \frac{1}{8}\left[18-\{1-(3-4 p) p+(1-p) \cos 2 r\} \cos \theta_{B}\right. \\
& \left.-2(1-p) \cos r\left\{2 \cos 2 \alpha_{B}\left(1+\cos \theta_{B}\right) \pm 5 \cos \theta_{B} \mp 5\right\}\right] .
\end{aligned}
$$

In Eq. (16), the upper sign in " $\pm "$ and in " $\mp "$ stands for Alice's payoff and the lower one for Bob's payoff. The results of Eq. (16) are, again, inverted to the results of Ref. 23] for $p=0$ and reduces to the result of [2] for setting both $r$ and $p$ equal to zero. Also, a close look on the equation immediately reveals that for a fully decohered game $(p=1)$, the effects of acceleration $r$ and quantum phase $\alpha_{B}$ become irrelevant. Under such condition $P_{A, B}^{Q \theta_{B}}=1 / 8\left(18-2 \cos \theta_{B}\right)$ that results in large payoffs when Bob executes $\hat{D}$. Similarly, for $p<1$, the strategy profiles $(\hat{Q}, \hat{C})$ and $(\hat{Q}, \hat{Q})$ are two equilibrium outcomes (at which $P_{A}^{Q C}=P_{B}^{Q C}$ and $\left.P_{A}^{Q Q}=P_{B}^{Q Q}\right)$. However, for strategy profile $(\hat{Q}, \hat{C})$ the payoffs increases with increasing value of $p$ whereas for strategy profile $(\hat{Q}, \hat{Q})$ it decreases. As stated above, against Bob's strategy $\hat{C}$ Alice's best strategy is $\hat{D}$ and against Bob's strategy $\hat{D}$ Alice's best strategy is $\hat{Q}$. This means that no single strategy of Alice dominate her against every strategy of Bob. The payoff matrix is symmetric from the perspective of strategy $\hat{Q}$. The strategy profile $(\hat{Q}, \hat{C})$ (and hence $(\hat{C}, \hat{Q}))$ is Pareto inefficient and the strategy profile $(\hat{Q}, \hat{Q})$ is both Pareto optimal and Nash equilibrium.

Finally, we investigate the effects of decoherence and acceleration on the payoffs when one player is limited to the classical strategic space and the other is allowed to choose any move from the quantum mechanical strategic space. This choice of the strategic spaces make the game unfair both in inertial and noninertial setups. In inertial setup, it is shown [2] that there exists a move for the quantum player to outsmart the classical player regardless of what the classical player is choosing from his strategic space. This move is known, in the literature on quantum games, as the miracle move and is given by

$$
\hat{M}=\hat{U}\left(-\frac{\pi}{2}, \frac{\pi}{2}\right)=\frac{i}{\sqrt{2}}\left(\begin{array}{cc}
-1 & 1 \\
1 & 1
\end{array}\right) .
$$

In noninertial frame, it is shown in 23 that playing this move always results in a poor result for the quantum player. This result is contradictory to the quantum mechanical results obtained in inertial setup of the game. The disagreement between the results of the game in the two setups, as discussed earlier, is because of the choice of choosing the operator $\hat{D}$ for constructing the entangling operator $\hat{J}$. 


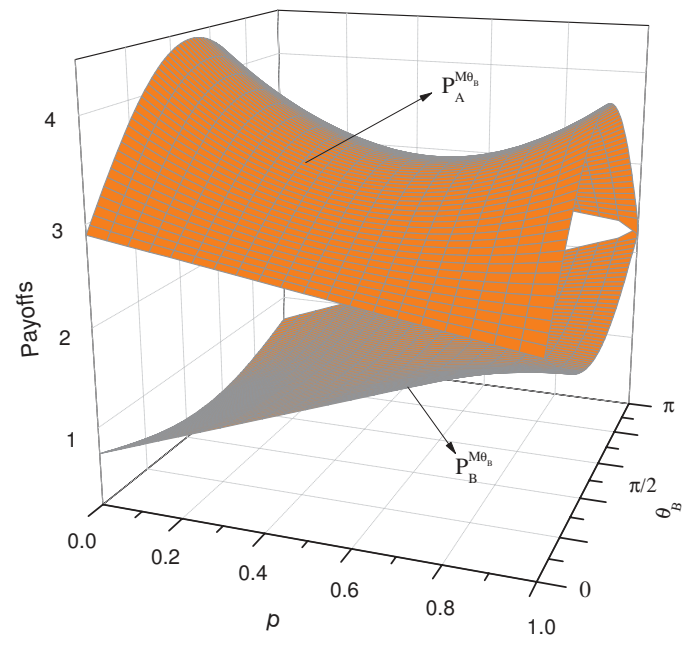

Figure 3: (color online) The payoffs of the players are plotted against the decoherence parameter $p$ and $\theta_{B}$ when Alice plays the miracle move $\hat{M}$. The acceleration parameter $r=\pi / 6$.

To study the effects of miracle move $\hat{M}$ in the present setup of the game, we restrict Bob to classical strategic space and allow Alice to execute $\hat{M}$. The payoffs under this condition become

$$
\begin{aligned}
P_{A}^{M \theta_{B}}= & \frac{1}{4}\left[\frac{7}{2}\{1+p(4 p-3)+(1-p) \cos 2 r\} \sin \theta_{B}\right. \\
& \left.+(1-p) \cos r\left(\sin \theta_{B}+3\right)+9\right], \\
P_{B}^{M \theta_{B}}= & \frac{1}{4}\left[-\frac{3}{2}\{1+p(4 p-3)+(1-p) \cos 2 r\} \sin \theta_{B}\right. \\
& \left.+(1-p) \cos r\left(\sin \theta_{B}-7\right)+9\right] .
\end{aligned}
$$

It can be seen from Eq. (18) that setting $p=0$ does not produce the result of [23], rather the results are inverted. However, setting $p=r=0$ produce the results of 22. The payoff matrix of Eq. (18) is symmetric with respect to the interchange of the players. It can be checked that for noiseless game, $P_{A}^{M \theta_{B}}>$ $P_{B}^{M \theta_{B}}$ for Bob's classical strategic space. That is, the quantum player dominates the classical one in the noiseless relativistic setup of the game by playing $\hat{M}$. In fact, as can be seen from Eq. (18), the choice from the two classical moves is meaningless against $\hat{M}$ both for noisy and noiseless setup of the game. However, 
there is a remarkable difference between the noisy and noiseless game. That is, for a fully decohered game $(p=1) P_{A}^{M \theta_{B}}=P_{B}^{M \theta_{B}}=2.25$, irrespective of the choice of Bob $\left(\theta_{B}=0\right.$ or $\left.\pi\right)$ and of acceleration of the frame. Thus for a fully decohered game the unfair game just become a fair game that always ends up in a single outcome to both players, provided one player is playing the miracle move and the other is limited to the classical two strategy space. Bob cannot outscore Alice even if he has access to the entire classical space spanned by $\theta_{B}$ for the whole range of decoherence parameter $p$ and acceleration parameter $r$. Figure 3 shows that how the payoffs of the players varies against $p$ and $\theta_{B}$. One can see that against Alice strategy $\hat{M}$ the best that Bob can gain is to play either $\hat{C}$ or $\hat{D}$. These results show that the miracle move is a special move in every setup of the game. In noiseless inertial and noninertial setups, it always let the player win. Against the two classical strategies, for a fully decohered case it makes the game fair and equally benefits the two players.

\section{Summary}

The effect of decoherence by using amplitude damping channel on the payoffs function of the players in the quantum prisoners' dilemma in noninertial frame has been investigated. It is shown that for unentangled initial state,generally, no strategy profile results in equal payoffs to both players and the game becomes an asymmetric game. Interestingly enough, the symmetric classical payoff matrix is recovered regardless of the acceleration of the frame for a fully decohered game. The local environment of the inertial player has no effect on the payoff functions of the players. The biasing effect of the acceleration of the noninertial frame is compensated by the amplitude damping noise. The inertial player always outscores the noninertial player by playing $\hat{D}$.

For maximally entangled initial state, it is shown that when the players are bound to the classical strategic spaces, the payoff matrix is a symmetric payoff matrix and the strategy profiles $(\hat{C}, \hat{C})$ and $(\hat{D}, \hat{D})$ become Pareto inefficient and there is no dilemma left in the game provided the game is fully decohered. For other values of decoherence parameter $p$, the strategy profile $(\hat{C}, \hat{C})$ is Pareto Optimal and $(\hat{D}, \hat{D})$ is Nash equilibrium which makes the strategy $\hat{D}$ a dominant strategy. The payoff matrix is symmetrical with respect to quantum strategy $\hat{Q}$ and the dominant strategy against $\hat{D}$ is the quantum strategy $\hat{Q}$. The strategy profile $(\hat{Q}, \hat{Q})$ is both Pareto optimal and Nash equilibrium. It is shown that the two classical moves become irrelevant against the miracle move $\hat{M}$, that is, both $\hat{C}$ and $\hat{D}$ lead to the same poor payoff for classical player. However against $\hat{C}$ and $\hat{D}$, for a fully decohered game, the miracle move reduce the game to a single outcome where both players are equally benefited and thus throw out the unfairness that exists in both inertial and noninertial setup of the quantum form of the game. The miracle move proves itself to be treated as a special move under any setup of the game. 


\section{References}

[1] Meyer, D. A.: Quantum Strategies. Phys. Rev. Lett. 82 1052-1055 (1999)

[2] Eisert, J., Wilkens, J., Lewenstein, M.: Quantum Games and Quantum Strategies. Phys. Rev. Lett. 83, 3077-3080 (1999)

[3] Marinatto, L., Weber, T.: A quantum approach to static games of complete information. Phys. Lett. A 272, 291-303 (2000)

[4] Li, H., Du, J., Massar, S.: Continuous-Variable Quantum Games. Phys. Lett. A 306 73-78 (2002)

[5] Lo, C. F., Kiang, D.: Quantum Bertrand duopoly with differentiated products. Phys. Lett. A 321 94-98 (2004)

[6] Flitney, A. P., Abbott D.:Quantum version of the Monty Hall problem. Phys. Rev. A 65, 062318 (2002)

[7] Iqbal, A., Toor, A. H.: Backwards-induction outcome in a quantum game. Phys. Rev. A 65, 052328 (2002)

[8] Flitney, A. P., Ng, J., Abbott, D.: Quantum Parrondo's games. Physica A $31435-42(2002)$

[9] Goldenberg, L., Vaidman, L., Wiesner, S.: Quantum Gambling. Phys. Rev. Lett. 82, 3356-3359 (1999)

[10] Khan, S., Ramzan, M., Khan, M. K.: Quantum Model of Bertrand Duopoly. Chin. Phys. Lett. 27, 080302 (2010)

[11] Chen, L. K., Ang, H., Kiang, D., Kwek. L.C. Lo, C. F.: Quantum prisoner dilemma under decoherence. Phys. Lett. A 316 317-323 (2003)

[12] Flitney, A. P., Abbott, D.: Quantum games with decoherence. J. Phys. A: Math. Gen. 38 449-459 (2005)

[13] Khan, S., Ramzan, M., Khan, M. K.: Quantum Parrondo's Games Under Decoherence Int. J. Theo. Phys. 49 31-41 (2010)

[14] Alsing, P. M., Fuentes-Schuller, I., Mann, R. B., Tessier, T. E.: Entanglement of Dirac fields in noninertial frames. Phys. Rev. A 74, 032326 (2006)

[15] Ling, Y., He, S., Qiu, W., Zhang, H.: Quantum entanglement of electromagnetic field in non-inertial reference frames.J. Phys. A: Math. Theor. 40 9025-9032 (2007)

[16] Gingrich, R. M., Adami, C.: Quantum Entanglement of Moving Bodies. Phys. Rev. Lett. 89, 270402 (2002)

[17] Pan, Q., Jing, J.: Degradation of nonmaximal entanglement of scalar and Dirac fields in noninertial frames. Phys. Rev. A 77, 024302 (2008) 
[18] Fuentes-Schuller, I., Mann, R. B.: Alice Falls into a Black Hole: Entanglement in Noninertial Frames. Phys. Rev. Lett. 95, 120404 (2005)

[19] Terashima, H., Ueda, M.: Relativistic Einstein-Podolsky-Rosen correlation and Bell's inequality. Int. J. Quantum Inf. 1, 93-114 (2003)

[20] Khan, S., Khan, M. K.: Open quantum systems in noninertial frames. J. Phys. A: Math. Theor. 44045305 (2011)

[21] wang J and Jing J.: Quantum decoherence in noninertial frames. Phys. Rev. A 82, 032324 (2010)

[22] Khan, S.: Entanglement of tripartite states with decoherence in non-inertial frames. J. Mod. Opt. 59 250-258 (2012)

[23] Khan, S., Khan, M. K.: Relativistic quantum games in noninertial frames. J. Phys. A: Math. Theor. 44355302 (2011)

[24] Takagi, S.: Vacuum Noise and Stress Induced by Uniform Acceleration. Prog. Theor. Phys. Suppl. 88 1-142 (1986)

[25] Alsing, P. M., McMahon, D. Milburn, G. J.: Teleportation in a non-inertial frame. J. Opt. B: Quantum Semiclass. Opt. 6 S834-S843 (2004)

[26] Aspachs, M., Adesso, G., Fuentes, I.: Optimal Quantum Estimation of the Unruh-Hawking Effect. Phys. Rev. Lett. 105, 151301 (2010)

[27] Martin-Martinez E, Garay L J, and Leon J.: Quantum entanglement produced in the formation of a black hole. Phys. Rev. D 82, 064028 (2010).

[28] Bruschi, D. E., Louko, J., Martin-Martinez, E., Dragan, A., Fuentes, I.: Unruh effect in quantum information beyond the single-mode approximation. Phys. Rev. A 82, 042332 (2010)

[29] Davies, P. C. W.: Scalar production in Schwarzschild and Rindler metrics. J. Phys. A 8 609-616 (1975)

[30] Unruh, W. G.: Notes on black-hole evaporation. Phys. Rev. D 14, 870-892 (1976)

[31] Benjamin, S. C., Hayden, P. M.: quantum games and quantum strategies. Phys. Rev. Lett. 8706980 (2001)

[32] Flitney, A. P., Hollenberg, L. C. L.: Nash equilibria in quantum games with generalized two-parameter strategies. Phys. Lett. A 363 381-388 (2007) 